\title{
Current sheets at low altitudes in the Martian magnetotail
}

\author{
J. S. Halekas, ${ }^{1}$ D. A. Brain, ${ }^{1}$ R. J. Lillis, ${ }^{1}$ M. O. Fillingim,,${ }^{1}$ D. L. Mitchell, ${ }^{1}$ and R. P. $\operatorname{Lin}^{1}$ \\ Received 7 March 2006; revised 3 May 2006; accepted 17 May 2006; published 4 July 2006.
}

[1] We present observations of 1116 current sheet crossings in the Martian magnetotail during one Martian year, as identified from magnetic field rotations measured by Mars Global Surveyor (MGS) at $\sim 400 \mathrm{~km}$ altitude and 2 am local time. Crossings are observed everywhere except above strong crustal fields, and many occur in clusters, with preferred locations varying as a function of season and IMF draping direction. Magnetic polarities are consistent with day-side IMF draping directions and a two-lobe induced magnetotail. Energetic "plasma sheet" electrons are often absent, implying that currents can be carried by ions or lowenergy electrons, with thicknesses of $<\sim 100 \mathrm{~km}$ arguing for electrons. The presence of a thin current sheet at low altitudes, with specific seasons and IMF directions favored for some geographic locations, implies that reconnection between crustal fields and the draped IMF may play an important role in its formation and location. Citation: Halekas, J. S., D. A. Brain, R. J. Lillis, M. O. Fillingim, D. L. Mitchell, and R. P. Lin (2006), Current sheets at low altitudes in the Martian magnetotail, Geophys. Res. Lett., 33, L13101, doi:10.1029/2006GL026229.

\section{Introduction}

[2] The plasma environment of a planet with no global magnetic field is fundamentally different from the terrestrial magnetosphere. Solar wind plasma interacts directly with the atmosphere, mass-loading the solar wind, slowing and deflecting the flow, and draping magnetic field lines around the ionosphere. However, as field lines drape around the planet, they form an elongated two-lobed structure with a central current sheet behind the planet, similar to the geomagnetic tail.

[3] Current sheets have been observed in the induced magnetotail of Mars (and also Venus) [Dubinin et al., 1991; Luhmann et al., 1991, McComas et al., 1986], at altitudes of thousands of km, far enough from Mars that the influence of the strong crustal fields [Acuña et al., 1999] is small. The upstream interplanetary magnetic field (IMF) clock angle controls the orientation of tail lobes and current sheet, consistent with IMF draping around Mars [Yeroshenko et al., 1990].

[4] Recent observations from Mars Global Surveyor (MGS) show that induced lobe magnetic fields and current sheets can be observed at the $\sim 400 \mathrm{~km}$ MGS mapping orbit [Ferguson et al., 2005]. This demonstrates that, even at low altitude where the effects of crustal fields are important, induced tail fields still play a significant role. Indeed, we will find that we observe many current sheet crossings, even

\footnotetext{
${ }^{1}$ Space Sciences Laboratory, University of California, Berkeley, California, USA.
}

Copyright 2006 by the American Geophysical Union. 0094-8276/06/2006GL026229\$05.00 at the low MGS orbital altitude, and that the orientation of these current sheets is compatible with a two-lobe induced magnetotail.

\section{Example Current Sheet Crossing}

[5] Figure 1 shows a typical nightside current sheet crossing. Magnetic fields are shown in MSO (Mars Solar Orbital) coordinates, with the X-axis toward the sun, the Zaxis perpendicular to Mars' orbital plane, and the Y-axis therefore nearly anti-parallel to Mars' orbital velocity. Comparison with the FSU90 crustal field model [Cain et al., 2003] (top) shows clearly that the field rotation is not associated with crustal fields, though significant crustal fields are seen before (north of) the crossing. A $B_{x}$ reversal, concurrent with near zero $B_{y}$ and $B_{z}$, is consistent with a transition between the two induced tail lobes.

[6] High fluxes of electrons with energies below $1 \mathrm{keV}$ are seen at the crossing, analogous to the terrestrial plasma sheet. Pitch angle measurements show that this population is nearly isotropic, though field-aligned flows toward Mars exist before the crossing. Electrons may be accelerated by tailward $\vec{J} \times \vec{B}$ magnetic tension forces [Dubinin et al., 1993] or gain energy from the convection electric field by executing trapped non-adiabatic orbits in the neutral sheet [Ip, 1992].

[7] We calculate the current sheet normal using the wellknown minimum variance analysis introduced by Sonnerup and Cahill [1967], and find a normal of [0.15, 0.87, -0.47]. The inferred geometry is shown in the inset in Figure 1. The ratio between the second and third eigenvalues is 22.4 , indicating that the normal direction is well defined. MGS travels a linear distance of $200 \mathrm{~km}$ across the current sheet, which lies at an oblique angle to the orbit. The dot product between the MGS velocity vector and the current sheet normal is 0.223 , implying a $45 \mathrm{~km}$ thick sheet. Previous observations at Mars and Venus found thicknesses of 500 to several thousand km [Dubinin et al., 1993; McComas et al., 1986; Dubinin et al., 1991; Luhmann et al., 1991]; however, these observations were also at much higher altitudes. The magnetic field jump of $\sim 28 \mathrm{nT}$ implies a current density of $\sim 0.5 \mu \mathrm{A} / \mathrm{m}^{3}$.

\section{Current Sheet Distribution}

[8] We searched the first Martian year of MGS nightside mapping data (June 1999 through April 2001) and found 1116 current sheet crossing. We found crossings by examining magnetic field components, and also magnetic field residuals with respect to the FSU90 model [Cain et al., 2003], and searching for sharp reversals in the $B_{x}$ component at times when $B_{y}$ and $B_{z}$ were small compared to $B_{x}$. By looking at residuals, we were able to find some current sheets even in regions of moderate crustal fields. We 


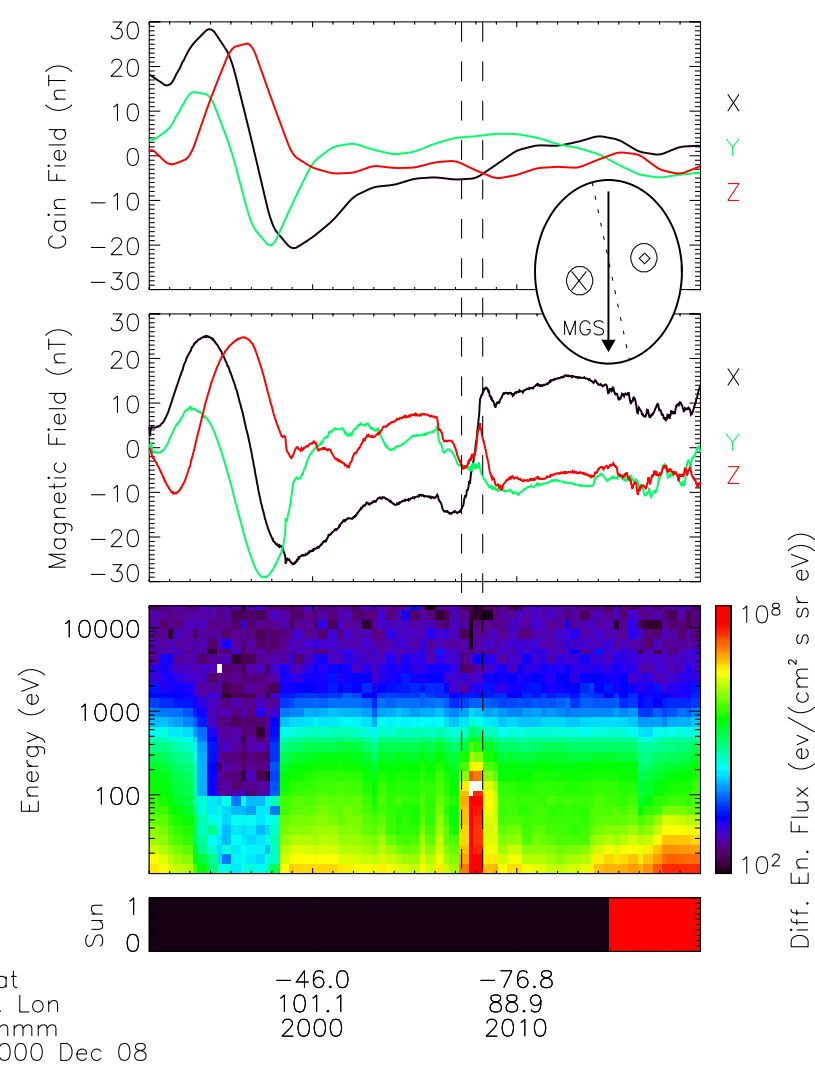

Figure 1. Current sheet crossing at 20:08 UT on Dec. 8, 2000, with magnetic field in MSO coordinates. The approximate extent of the current sheet is bracketed by vertical dashed lines. (top) FSU90 model fields are shown for comparison. The inferred geometry as viewed from the tail is shown in the inset, with the solid arrow showing the MGS orbit trajectory, the dashed line showing the current sheet, and symbols indicating magnetic field polarities.

identified crossings on $\sim 13 \%$ of MGS orbits. A few orbits have multiple crossings, perhaps due to bifurcation or motion of the current sheet, but this is very rare.

[9] Figure 2 shows histograms of dayside draping direction, as defined by Brain et al. [2006a], for two magnetic polarities. Draping direction is defined so that $0^{\circ}$ corresponds to local eastward draping on the dayside. If dayside draping direction corresponded perfectly with upstream IMF clock angle, as MGS orbits from north to south on the night side, we would cross from $-B_{x}$ lobe to $+B_{x}$ lobe for draping directions of $0-180^{\circ}$ (some northward component), and from $+B_{x}$ to $-B_{x}$ for draping directions of $180-$ $360^{\circ}$. In fact, the two histograms shown in Figure 2 conform fairly well with expectations, though the dayside draping direction does not correspond perfectly to upstream clock angle (likely because of "weathervaning" as field lines are dragged through the ionosphere [Brain et al., 2006a]. This demonstrates that the observed magnetic field polarities are consistent with crossings of a current sheet between two induced tail lobes.

[10] Figure 3a shows current sheet crossings (white symbols) in planetary coordinates and the total crustal field at MGS altitude from the FSU90 model [Cain et al., 2003]. Crossings can be observed everywhere except regions of strong crustal fields, implying that current sheets there are

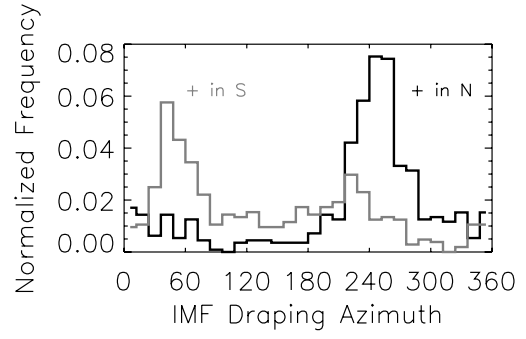

Figure 2. Histograms of IMF draping direction for current sheet crossings with positive $B_{x}$ component before (north of, black) and after (south of, grey) crossings. Consistently normalized so that the integral of the sum of the two distributions is unity.

pushed to higher altitudes, above MGS. This is consistent with model results, where the transition between lobes can be found above a closed magnetic field loop on the night side [see Ma et al., 2004, Figure 3]. Even in regions of weak crustal fields we find clear clusters of crossings, so crustal fields do not completely explain the distribution.
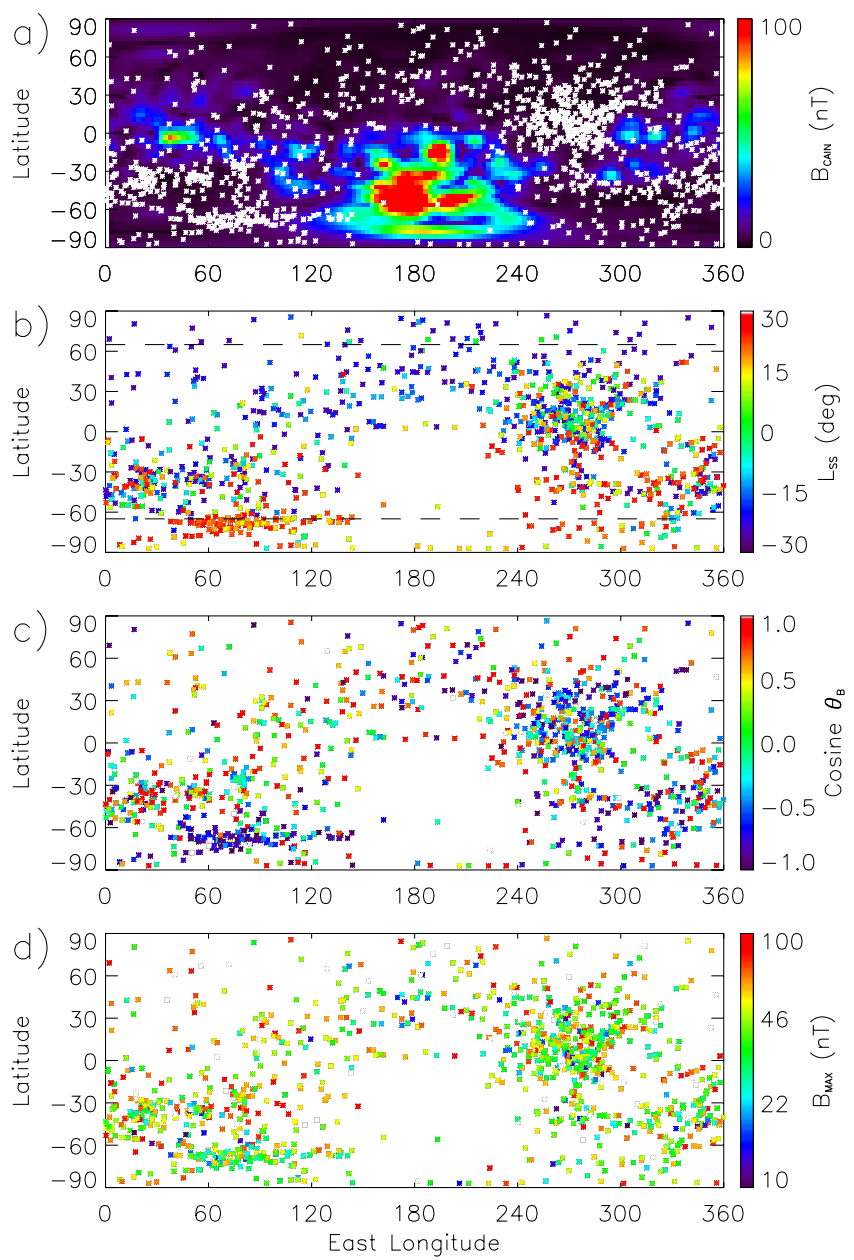

Figure 3. Current sheet crossings in geographic coordinates, plotted over crustal magnetic field at (a) orbital altitude (FSU90 model), (b) color coded by subsolar latitude, (c) cosine of IMF draping direction, and (d) pressure proxy. 

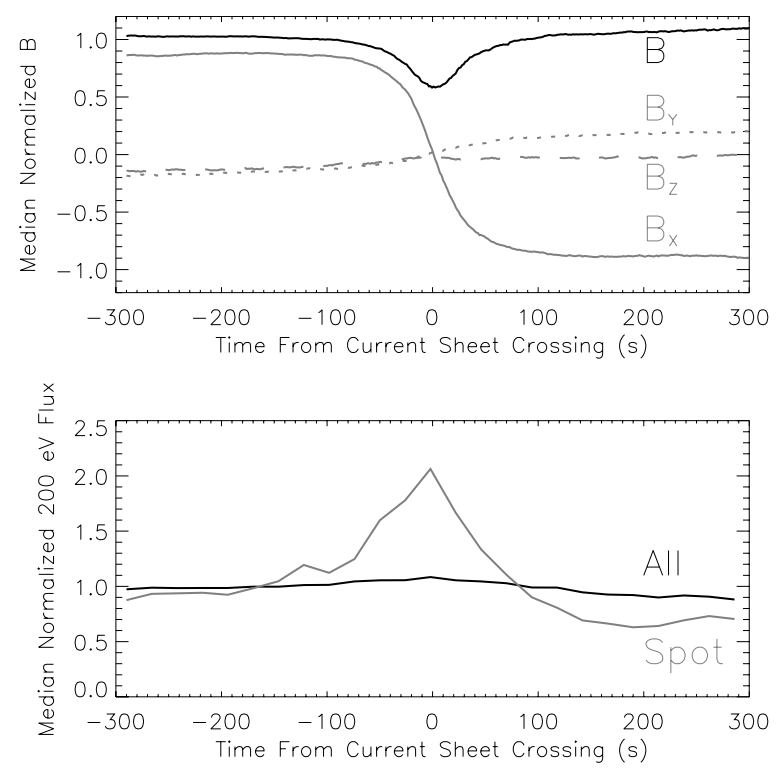

Figure 4. Median normalized magnetic field and electron flux for current sheet crossings. Median flux also shown for "spot" extending from $40-140^{\circ} \mathrm{E}, 60-80^{\circ} \mathrm{S}$.

[11] Figure 3b shows crossings with color corresponding to subsolar latitude $L_{S S}$, demonstrating a seasonal dependence. In general, one expects to see more crossings in the northern hemisphere for negative $L_{S S}$ (and vice versa for positive $L_{S S}$ ) since that hemisphere will be tilted toward the center of the tail. For the same reason, at high latitudes, the full range of $L_{S S}$ is not available on the night side, and the extent of these regions are indicated by the dashed lines in Figure $3 \mathrm{~b}$. However, even beyond this expected asymmetry, we observe clusters of crossings during certain seasons. Note especially the two nearby clusters $\left(0-90^{\circ} \mathrm{E}, 30-60^{\circ} \mathrm{S}\right.$ and $\left.40-140^{\circ} \mathrm{E}, 60-80^{\circ} \mathrm{S}\right)$ in the southwest quadrant, with very different preferred $L_{S S}$. Part of this difference is due to a selection bias, since large negative $L_{S S}$ cannot be observed on the night side at high southern latitudes. However, the noted clusters also have significantly different $L_{S S}$ than other regions at the same latitudes.

[12] In Figure 3c, we also find a clear dependence on IMF draping direction. Again, the two clusters referenced above differ, with dawnward IMF draping strongly preferred in the southernmost cluster, and duskward draping in the other. Reconnection with nearby crustal fields may be important, with specific IMF polarities thereby selected in certain regions.

[13] Finally, in Figure 3d, we find no clear dependence on the solar wind dynamic pressure proxy defined by Brain et al. [2005].

[14] In general, though the preferred location of observed current sheets changes, the absolute likelihood of observing current sheet crossings does not depend strongly on any of the above parameters. On orbits with crossings we find a moderate tendency for IMF draping opposite the "clustered direction" [Brain et al., 2006a], and a slightly higher average pressure proxy. We also find no clear organization by MSO coordinates, though we are limited by the fixed 2 am local time of the MGS orbit on the night side. We also find no clear organization by the convection electric field direction like that found at higher altitudes and in models [Federov et al., 2006; Modolo et al., 2005]. However, it is unlikely that this asymmetry would emerge clearly at low altitude.

\section{Current Sheet Morphology}

[15] In Figure 4 we show median magnetic field and electron flux for all 1116 current sheet crossings. Magnetic field magnitude and components are normalized by the average pre-crossing magnitude before taking medians, and for current sheets with $-B_{x}$ in the north all components are pre-inverted. MGS is always at 2 am (and therefore negative $\mathrm{y}$ ), and the median orientation is such that $+B_{x}$ is associated with $-B_{y}$, and vice versa. The field on both sides of the current sheet is nearly parallel to the $\mathrm{X}$-axis, but drapes slightly toward the $\mathrm{X}-\mathrm{Z}$ plane with increasing distance downstream. This orientation is consistent with highly draped magnetic field lines which drag through the ionosphere, slip around the sides of the planet, and then are pulled toward the center of the tail by magnetic tension forces. We show this inferred magnetic field geometry in Figure 5.

[16] The average current sheet thickness is at most a few hundred $\mathrm{km}$ (corresponding to the $\sim 100 \mathrm{~s}$ crossing duration, and the MGS orbital velocity of $\sim 3 \mathrm{~km} / \mathrm{s}$ ); however, in general the thickness is even less since we may cross the current sheet obliquely, as in our example case. The current sheet may be so thin because MGS is close to Mars and field lines are still extremely kinked from being dragged through the ionosphere. Alternatively, a thin current sheet may reveal recent reconnection with crustal fields. The current sheet thickness is small compared to typical ion gyroradii in the Martian system, implying that electron dynamics may dominate.

[17] Median electron flux, pre-normalized by the average value before the crossing, is shown for all crossings in Figure 4, and for the cluster at $40-140^{\circ} \mathrm{E}, 60-80^{\circ} \mathrm{S}$. The average flux increase for all current sheets is only on the order of $5-10 \%$, while flux more than doubles in the "spot". For some observations, fluxes are so high that the MGS electron instrument saturates, and these are not included here, implying that the real median flux is even larger than shown. This suggests some special property of this particular cluster of crossings. Enhanced reconnection may be favored when the strong southern anomalies are at dawn and the IMF draping direction is dawnward (as

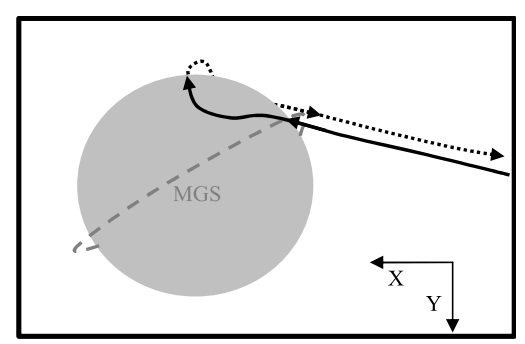

Figure 5. Cartoon showing magnetic topology inferred from median magnetic field components observed by MGS at current sheet crossings. Magnetic field line is solid north of the current sheet and dotted south of it. 
in this case), as predicted by Harnett and Winglee [2005]. We note that this "spot" also has a high concentration of peaked electron spectra, possibly implying acceleration in parallel electric fields [Brain et al., 2006b].

[18] The small median flux increase for all crossings may imply that most of the cross-tail current for many low altitude cases is carried by either ions or low-energy electrons, with a thin current sheet arguing for the latter. The requisite current density could be carried by low-energy electrons with sufficiently high densities. For example, for a density of $10 \mathrm{~cm}^{-3}$, a cross-tail drift velocity equivalent to an energy offset of $0.27 \mathrm{eV}$ would explain the current density we found for our example crossing. Electron densities this high at $400 \mathrm{~km}$ altitude, however, may suggest either access for dayside ionospheric plasma via draped magnetic field lines, or the presence of a minimal nightside ionosphere.

\section{Conclusions}

[19] We observe many current sheet crossings, even at the low MGS orbital altitude. Magnetic polarities are consistent with dayside IMF draping directions and a classic two-lobe induced magnetotail structure. Crustal magnetic fields push current sheets above MGS altitude, and changing season and IMF draping direction affect the location of current sheet crossings, possibly highlighting the importance of reconnection with nearby crustal fields. Magnetic field orientations are consistent with draped field lines which slip around the planet and are pulled toward the center of the tail by magnetic tension forces. The extremely thin current sheet implies that electron dynamics likely dominate at low altitudes, and the lack of a consistent increase in higher-energy electrons implies that low-energy electrons may often carry most of the cross-tail current. These observations demonstrate that the tail field is highly draped near Mars, and provide hints of the possible importance of reconnection between draped solar wind fields and crustal fields.

[20] Acknowledgment. This work was supported by NASA grant NNG05GJ24G. We thank two reviewers for helpful suggestions.

\section{References}

Acuña, M. H., et al. (1999), Global distribution of crustal magnetization discovered by the Mars Global Surveyor MAG/ER experiment, Science, $284,790-793$.
Brain, D. A., J. S. Halekas, R. J. Lillis, D. L. Mitchell, R. P. Lin, and D. H. Crider (2005), Variability of the altitude of the Martian sheath, Geophys. Res. Lett., 32, L18203, doi:10.1029/2005GL023126.

Brain, D. A., D. L. Mitchell, and J. S. Halekas (2006a), The magnetic field draping direction at Mars from April 1999 through August 2004, Icarus, 182(2), 464-473, doi:10.1016/j.icarus.2005.09.023.

Brain, D. A., J. S. Halekas, L. M. Peticolas, R. P. Lin, J. G. Luhmann, D. L. Mitchell, G. T. Delory, S. W. Bougher, M. H. Acua, and H. Rme (2006b), On the origin of aurorae on Mars, Geophys. Res. Lett., 33, L01201, doi:10.1029/2005GL024782.

Cain, J. C., B. B. Ferguson, and D. Mozzoni (2003), An $\mathrm{n}=90$ internal potential function of the Martian crustal magnetic field, J. Geophys. Res., 108(E2), 5008, doi:10.1029/2000JE001487.

Dubinin, E., R. Lundin, W. Riedler, K. Schwingenschuh, J. G. Luhmann, C. T. Russell, and L. H. Brace (1991), Comparison of observed plasma and magnetic field structures in the wakes of Mars and Venus, J. Geophys. Res., $96,11,189-11,197$

Dubinin, E., R. Lundin, O. Norberg, and N. Pissarenko (1993), Ion acceleration in the Martian tail: Phobos observations, J. Geophys. Res., 98 , $3991-3997$

Federov, A., et al. (2006), Structure of the martian wake, Icarus, 182(2), 329-336, doi:10.1016/j.icarus.2005.09.021.

Ferguson, B. B., J. C. Cain, D. H. Crider, D. A. Brain, and E. M. Harnett (2005), External fields on the nightside of Mars at Mars Global Surveyor mapping altitudes, Geophys. Res. Lett., 32, L16105, doi:10.1029/ 2004GL021964.

Harnett, E. M., and R. M. Winglee (2005), Three-dimensional fluid simulations of plasma asymmetries in the Martian magnetotail caused by the magnetic anomalies, J. Geophys. Res., 110, A07226, doi:10.1029/ 2003JA010315.

Ip, W.-H. (1992), Ion acceleration at the current sheet of the Martian magnetosphere, Geophys. Res. Lett., 19, 2095-2098.

Luhmann, J. G., C. T. Russell, K. Schingenschuh, and Ye. Yeroshenko (1991), A comparison of induced magnetotails of planetary bodies: Venus, Mars, and Titan, J. Geophys. Res., 96, 11,199-11,208.

Ma, Y., A. F. Nagy, I. V. Sokolov, and K. C. Hansen (2004), Threedimensional, multispecies, high spatial resolution MHD studies of the solar wind interaction with Mars, J. Geophys. Res., 109, A07211, doi:10.1029/2003JA010367.

McComas, D. J., H. E. Spence, C. T. Russell, and M. A. Saunders (1986), The average magnetic field draping and consistent plasma properties of the Venus magnetotail, J. Geophys. Res., 91, 7939-7953.

Modolo, R., G. M. Chanteur, E. Dubinin, and A. P. Matthews (2005), Influence of the solar EUV flux on the Martian plasma environment, Ann. Geophys., 23, 433-444.

Sonnerup, B. U. Ö, and L. J. Cahill Jr. (1967), Magnetopause structure and attitude from Explorer 12 observations, J. Geophys. Res., 72, 171-183.

Yeroshenko, Ye., W. Riedler, K. Schwingenschuh, J. G. Luhmann, M. Ong, and C. T. Russell (1990), The magnetotail of Mars: Phobos observations, Geophys. Res. Lett., 17, 885-888.

D. A. Brain, M. O. Fillingim, J. S. Halekas, R. J. Lillis, R. P. Lin, and D. L. Mitchell, Space Sciences Laboratory, University of California, Berkeley, 7 Gauss Way, Berkeley, CA 94720-7450, USA. (jazzman@ ssl.berkeley.edu) 